\title{
Article
}

\section{Energetic particle transport across the mean magnetic field: before diffusion}

Laitinen, Timo Lauri mikael and Dalla, Silvia

Available at https://clok.uclan.ac.uk/17565/

Laitinen, Timo Lauri mikael orcid iconORCID: 0000-0002-7719-7783 and Dalla, Silvia orcid iconORCID: 0000-0002-7837-5780 (2017) Energetic particle transport across the mean magnetic field: before diffusion. The Astrophysical Journal, 834 (2). p. 127. ISSN 0004-637X

It is advisable to refer to the publisher's version if you intend to cite from the work. http://dx.doi.org/10.3847/1538-4357/834/2/127

For more information about UCLan's research in this area go to

http://www.uclan.ac.uk/researchgroups/ and search for < name of research Group>.

For information about Research generally at UCLan please go to http://www.uclan.ac.uk/research/

All outputs in CLoK are protected by Intellectual Property Rights law, including Copyright law. Copyright, IPR and Moral Rights for the works on this site are retained by the individual authors and/or other copyright owners. Terms and conditions for use of this material are defined in the policies page.

\section{CLoK}

Central Lancashire online Knowledge www.clok.uclan.ac.uk

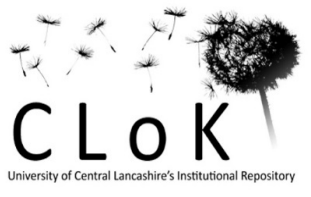


ENERGETIC PARTICLE TRANSPORT ACROSS THE MEAN MAGNETIC FIELD: BEFORE DIFFUSION

\author{
T. LAitinen AND S. Dalla \\ Jeremiah Horrocks Institute, University of Central Lancashire, Preston, UK; tlmlaitinen@uclan.ac.uk \\ Received 2016 July 7; revised 2016 November 1; accepted 2016 November 14; published 2017 January 9
}

\begin{abstract}
Current particle transport models describe the propagation of charged particles across the mean field direction in turbulent plasmas as diffusion. However, recent studies suggest that at short timescales, such as soon after solar energetic particle (SEP) injection, particles remain on turbulently meandering field lines, which results in nondiffusive initial propagation across the mean magnetic field. In this work, we use a new technique to investigate how the particles are displaced from their original field lines, and we quantify the parameters of the transition from field-aligned particle propagation along meandering field lines to particle diffusion across the mean magnetic field. We show that the initial decoupling of the particles from the field lines is slow, and particles remain within a Larmor radius from their initial meandering field lines for tens to hundreds of Larmor periods, for $0.1-10 \mathrm{MeV}$ protons in turbulence conditions typical of the solar wind at 1 au. Subsequently, particles decouple from their initial field lines and after hundreds to thousands of Larmor periods reach time-asymptotic diffusive behavior consistent with particle diffusion across the mean field caused by the meandering of the field lines. We show that the typical duration of the prediffusive phase, hours to tens of hours for $10 \mathrm{MeV}$ protons in 1 au solar wind turbulence conditions, is significant for SEP propagation to 1 au and must be taken into account when modeling SEP propagation in the interplanetary space.
\end{abstract}

Key words: diffusion - magnetic fields - Sun: particle emission - turbulence

\section{INTRODUCTION}

The propagation of cosmic rays through the heliosphere is affected by the large-scale interplanetary magnetic field and the turbulent fluctuations superposed on it. Understanding the nature of the effect of these fields on particle transport is necessary, as we want to understand the sources and acceleration processes of different cosmic-ray populations.

The turbulent fluctuations in the interplanetary magnetic field can be considered as scattering agents for the cosmic rays, prompting the description of their propagation as random walk. Parker (1965) used this concept to describe the propagation of cosmic rays in the time-asymptotic limit as diffusion. Determining the connection between the turbulence properties and the diffusion coefficients, however, has proven to be a difficult task. Jokipii (1966) considered a quasi-linear approach, where the transport along the mean field direction was affected by fluctuations of the scale of the particle's Larmor radius, whereas the propagation across the mean field was caused by the random walk experienced by the magnetic field lines due to turbulent fluctuations. The field-line random walk model has since been extended to consider the compound effect of the particles scattering along the random-walking field lines (Matthaeus et al. 2003; Shalchi 2010; Ruffolo et al. 2012), and the most advanced models generally compare well with full-orbit simulations (Giacalone \& Jokipii 1999) and some cosmic-ray observations (Burger et al. 2000).

The particle cross-field diffusion has also been applied in modeling solar energetic particle (SEP) propagation in the heliosphere (e.g., Zhang et al. 2009; Dröge et al. 2010; He et al. 2011; Giacalone \& Jokipii 2012; Qin et al. 2013). Recent SEP observations, however, have proved difficult to reconcile with the models. The solar wind turbulence properties, measured by spacecraft (e.g., Burlaga \& Turner 1976; Bavassano et al. 1982) and coupled with theoretical and modeling work, suggest that parallel diffusion dominates over cross-field diffusion, with the diffusion coefficient ratio $\kappa_{\perp} / \kappa_{\|} \sim 0.01 \quad$ (e.g., Giacalone \& Jokipii 1999; Burger et al. 2000; Potgieter et al. 2014). However, fits of SEP intensity profiles performed with injection scenario and diffusion coefficients as free parameters suggest a considerably larger value, $\kappa_{\perp} / \kappa_{\|} \sim 0.1$ (Dresing et al. 2012; Dröge et al. 2014). On the other hand, the sharp dropouts observed in some SEP events (e.g., Mazur et al. 2000) have been considered as evidence of only negligible cross-field diffusion of SEPs (Dröge et al. 2010; Wang et al. 2014).

The problem of accounting for the observed fast cross-field propagation for SEPs was recently addressed by Laitinen et al. (2013). Using full-orbit particle simulations, they found that the initial cross-field propagation with respect to the mean field direction is not diffusive and can be described as field-aligned propagation of particles along stochastically meandering field lines. They concluded that for a uniform background magnetic field with turbulence parameters corresponding to solar wind conditions near Earth, $10 \mathrm{MeV}$ protons propagated to distances of $1 \mathrm{au}$ from the source, remaining bound to their meandering field lines over timescales of $6 \mathrm{hr}$. However, relative to the mean magnetic field direction, the meandering field lines spread the particles to a much wider cross-field extent than the asymptotic diffusion assumption. At later stage, the particles could be considered diffusive with respect to mean field direction only after $20 \mathrm{hr}$ from their injection.

The Laitinen et al. (2013) study thus indicated that for SEPs early in the event, the use of the diffusion description for particle cross-field propagation is invalid, and that only at longer timescales can its use be justified. The following questions then arise: When and how does the transition from nondiffusive to diffusive cross-field propagation take place, and how is the transition related to properties of the plasma turbulence? How do the particles decouple from the field lines?

The particle decoupling from field lines has been discussed previously in attempts to understand and develop a theory for the time-asymptotic cross-field diffusion of particles in 
turbulent magnetic fields (e.g., Qin et al. 2002; Matthaeus et al. 2009; Ruffolo et al. 2012). However, quantifying the process of the particles leaving their field lines presents several challenges. The field-line meandering is typically much faster than the decoupling of a particle from a field line (e.g., Fraschetti \& Jokipii 2011). Thus, a particle's displacement in the cross-field direction is a measure of the random walk of the magnetic field line, rather than the particle's random walk relative to the meandering field line. On the other hand, determination of the particle's position relative to its original field line suffers from the uncertainty due to the variation of the magnetic field within the particle's path of gyration.

In this work, we introduce a new technique to determine the cross-field displacement of a particle from the meandering magnetic field line it initially follows. We use the new technique, presented in Section 2, to quantify the process of particle decoupling from its initial field line and evaluate the contribution of the decoupling of the particle to the propagation of the particles across the mean magnetic field. In Section 3 we show that the particle propagation across the field can be divided into two separate diffusion ranges, which are separated by a transition range. In Section 4, we discuss the physical nature of the diffusion phases and the transition phase between them and compare our results with current particle transport theories. Finally, we draw our conclusions in Section 5.

\section{MODELS}

We simulate charged particles by integrating their full orbits in a magnetic field given by

$$
\boldsymbol{B}(x, y, z)=B_{0} \hat{z}+\delta \boldsymbol{B}(x, y, z),
$$

where $B_{0}$ is a constant background field, along the $z$-axis, and $\delta \boldsymbol{B}(x, y, z)$ a fluctuating field, consisting of slab and 2D components, with energy ratio 20\%:80\% between the components, and a broken Kolmogorov power-law spectrum, with

$$
\begin{gathered}
P_{\text {slab }}\left(k_{\|}\right)=\frac{\delta B_{\|}^{2}}{B^{2}} \frac{C_{\|}}{1+\left(k_{\|} L_{c}\right)^{5 / 3}} \\
P_{2 \mathrm{D}}\left(k_{\perp}\right)=\frac{\delta B_{\perp}^{2}}{B^{2}} \frac{C_{\perp}}{1+\left(k_{\perp} L_{c}\right)^{8 / 3}},
\end{gathered}
$$

where $\delta B_{\|}^{2}$ and $\delta B_{\perp}^{2}$ are the variances of the turbulence slab and $2 \mathrm{D}$ components, respectively, $L_{c}$ is the breakpoint scale of the turbulence, for which we use $L_{c}=2.15 R_{\odot}$ in our study, with $R_{\odot}$ the solar radius, and $C_{\|}$and $C_{\perp}$ are normalization constants (see, e.g., Giacalone \& Jokipii 1999). We use $B_{0}=5 \mathrm{nT}$, consistent with the magnetic field strength at 1 au. The fluctuating field is formed numerically as a sum of Fourier modes logarithmically spaced between wavenumbers $2 \pi /(1 \mathrm{au})$ and $2 \pi /\left(10^{-4} \mathrm{au}\right)$, with the method described by Giacalone \& Jokipii (1999). The turbulence amplitude is parameterized by the variance of the turbulence, $\delta B^{2}$, which is varied in this study, and the ratio between $\delta B_{\|}^{2}$ and $\delta B_{\perp}^{2}$, which is $20 \%: 80 \%$ (Gray et al. 1996) unless otherwise stated.

In this work, we are studying how the particles decouple from the turbulent magnetic field lines. To measure this, we introduce a new technique: we analyze the cross-field displacement of a particle that returns back to the plane normal to the mean magnetic field that it was injected at. The method is depicted in Figure 1: the particle is started at $\left(x_{0}, y_{0}, z_{0}\right)$ and

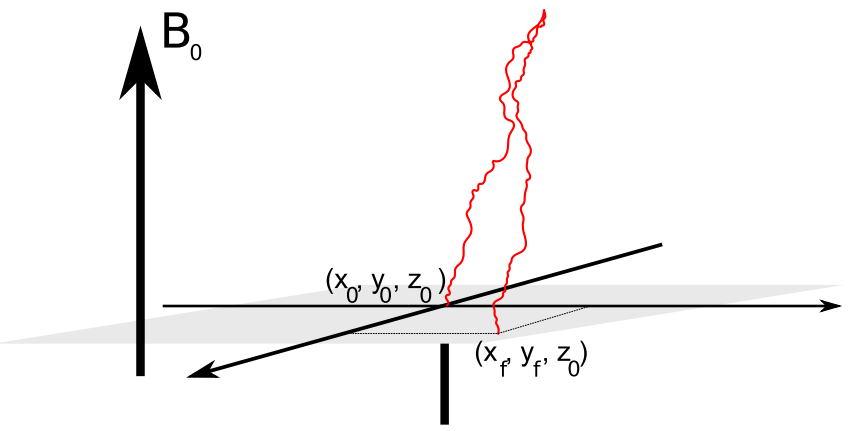

Figure 1. Schematic description of determining the cross-field displacement at $z=z_{0}$, with the particle's path shown by the red curve. The particle starts at $\left(x_{0}, y_{0}, z_{0}\right)$ and is followed until it reaches the $z=z_{0}$ plane again, at $\left(x_{f}, y_{f}, z_{0}\right)$.

traced until it returns to the $z=z_{0}$ plane, where its coordinates $\left(x_{f}, y_{f}, z_{0}\right)$ are recorded. A particle remaining perfectly on its field line would return within two Larmor radii of its starting point. To eliminate the displacement due to Larmor gyration, we calculate the particle's gyrocenter $\overline{\boldsymbol{r}}$ at the injection and return times, with

$$
\overline{\boldsymbol{r}}=\boldsymbol{r}+\frac{q}{|q| \Omega B} \boldsymbol{v} \times \boldsymbol{B}
$$

where $\boldsymbol{r}$ and $\boldsymbol{v}$ are the particle's position and velocity, respectively, and $q$ and $\Omega$ the particle charge and gyrofrequency. As our model of turbulence is axisymmetric, either $x$ or $y$ can be used as the representative direction perpendicular to the mean field. We calculate the displacement in the $x$ direction, defined as

$$
\Delta \bar{x}(t)^{2}=\left(\bar{x}_{f}-\bar{x}_{0}\right)^{2},
$$

where $t=t_{f}-t_{0}$ is the flight time of the particle, from the time of particle injection at $t_{0}$ to its return to the $z_{0}$ plane at $t_{f}$, and $\bar{x}_{0}$ and $\bar{x}_{f}$ are the $x$-coordinates of the particle's gyrocenter at the start of the simulation and when it returns to the $z=z_{0}$ plane, respectively. Defined in this way, $\Delta \bar{x}^{2}$ does not include the cross-field propagation of the particles directly due to the wandering of the field lines: were a particle to follow the meandering field line precisely, its gyrocenter would cross the starting plane at exactly the same location it started at, $\left(\bar{x}_{0}, \bar{y}_{0}, \bar{z}_{0}\right)$, resulting in $\Delta \bar{x}^{2}=0$.

\section{RESULTS}

To analyze the cross-field propagation of energetic particles, we studied the distribution of $\Delta \bar{x}^{2}\left(t_{f}\right)$, defined in Equation (5), within a monoenergetic population of particles. The particles are injected at random locations $\left(x_{0 i}, y_{0 i}, z_{0 i}\right)$ to minimize the possible effects of local structures in the generated turbulent magnetic fields. We ran simulations of typically 100,000 protons with isotropic pitch angle distribution in the $v_{z}>0$ hemisphere. The particles were propagated in a turbulent magnetic field until they returned back to initial plane, $z=z_{0 i}$. At the time of return, the square of the guiding center displacement, as given by Equation (5), was recorded.

We show an example of the simulation results in Figure 2, with a scatterplot of $\Delta \bar{x}^{2}$ as a function of the flight time $t$, for $10 \mathrm{MeV}$ protons, with $\delta B^{2} / B^{2}=0.316$. The time is normalized to the particle Larmor period, $T_{\mathrm{L}}=2 \pi / \Omega$. The median 


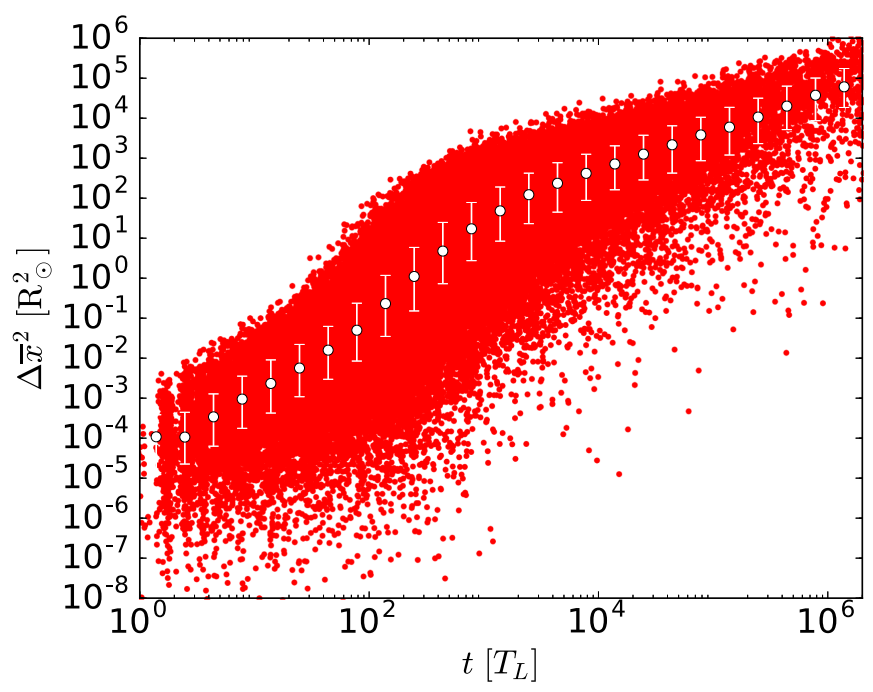

Figure 2. Displacement $\Delta \bar{x}^{2}$ of the returning particles as a function of time, in units $T_{\mathrm{L}}=2 \pi / \Omega$, for $10 \mathrm{MeV}$ protons, with $\delta B^{2} / B^{2}=0.316$. The red symbols depict the displacement of each simulated particle, and the white symbols the median displacement for different times. The error bars are drawn at lower and upper quartiles.

displacement for logarithmically spaced time ranges is shown by the white filled circles, and the lower and upper quartiles by the error bars. The Larmor radius of a $10 \mathrm{MeV}$ proton in the given magnetic field is $0.13 R_{\odot}$; thus, for the particles for which $\Delta \bar{x}^{2}<0.017 R_{\odot}^{2}$, the guiding center of the returning particle remains within a gyroradius of the initial location of the guiding center.

We can identify three time ranges of different behavior of $\Delta \bar{x}^{2}$ as a function of flight time. The first range, up to $t \sim 100 T_{\mathrm{L}}$, contains particles that return to the initial plane close to the original location and have roughly a linear trend of $\Delta \bar{x}^{2}$ as a function of time, consistent with a diffusive, or slightly superdiffusive, increase of the displacement. At around $t \sim 100 T_{\mathrm{L}}$, the spreading becomes faster, clearly superdiffusive, within the second range. The fast spreading continues until at $t \sim 1000 T_{\mathrm{L}}$ it relaxes back to a diffusive trend. For the purposes of this study, we name these ranges the first diffusion range, the transition range, and the second diffusion range.

In order to characterize the transition between the first and second diffusion ranges, we must determine when the transition takes place. To do this, we fit $\Delta \bar{x}^{2}$ as a function of time with a function that depicts initially a nondiffusive behavior, $\Delta \bar{x}^{2} \propto t^{\alpha}$, followed by a fast spreading across the field with $\Delta \bar{x}^{2} \propto t^{\beta}$ and a time-asymptotic diffusion, $\Delta \bar{x}^{2} \propto t$. Overall, the function has the form

$$
\Delta \bar{x}^{2}(t)=\Delta \bar{x}_{1}^{2}\left(\frac{t}{T_{\mathrm{L}}}\right)^{\alpha} \frac{1+\left(t / t_{1}\right)^{\beta-\alpha}}{1+\left(t / t_{2}\right)^{\beta-1}},
$$

where $\Delta \bar{x}_{1}^{2}$ represents the square of displacement at $t=T_{\mathrm{L}} \ll t_{1}<t_{2}$, and $t_{1}$ and $t_{2}$ are the start and end times of the transition range, respectively. At early times, $t \ll t_{1}$, the equation describes the first diffusion range, with

$$
\Delta \bar{x}^{2}(t) \stackrel{t \ll t_{1}}{\rightarrow} \Delta \bar{x}_{1}^{2}\left(\frac{t}{T_{\mathrm{L}}}\right)^{\alpha},
$$

whereas at late times, $t \gg t_{2}$, the second diffusion range is given as

$$
\Delta \bar{x}^{2}(t) \stackrel{t \gg t_{2}}{\rightarrow} \Delta \bar{x}_{2}^{2} \frac{t}{T_{\mathrm{L}}}
$$

where

$$
\Delta \bar{x}_{2}^{2}=\Delta \bar{x}_{1}^{2} \frac{t_{2}^{\beta-1}}{t_{0}^{\alpha-1} t_{1}^{\beta-\alpha}} .
$$

It should be noted that the form of the fit function has no physical justification as such. It is only used to trace the behavior of the particles in the three ranges.

We use Equation (6) to fit the median values of $\Delta \bar{x}^{2}$ as a function of time for different values of turbulence amplitude and particle energy, excluding times $t<5 T_{\mathrm{L}}$ from the fitting to avoid any potential initial non-gyrotropic effects. We choose median instead of mean as our statistics, as we are interested in the mechanism causing the transition and the time when it begins. A mean value is skewed toward large values due to individual particles having large displacements; thus, a mean displacement would represent the extent of the displacement rather than the behavior of the bulk of the particles.

In Figure 3, we show the results of our analysis of the displacement of $10 \mathrm{MeV}$ protons from their initial field lines in turbulent magnetic field with $\delta B^{2} / B^{2}=0.0316,0.0562,0.1$, 0.316 , and 0.562 , which represent the range of observed turbulence amplitudes at 1 au (e.g., Burlaga \& Turner 1976). In panel (a), we show the median displacements and the corresponding fits using Equation (6), as a function of time, in units $r_{\mathrm{L}}^{2}$, where $r_{\mathrm{L}}=v / \Omega$ is the particle's Larmor radius. The median values and fits show a three-regime structure observed in Figure 2 throughout the analyzed $\delta B^{2} / B^{2}$ range.

In Figure 3(b) we show $\Delta \bar{x}_{1}^{2}$ and $\Delta \bar{x}_{2}^{2}$, which quantify the rate of the displacement of the particles from their field lines in unit time $T_{\mathrm{L}}$ in the first and second diffusive ranges, respectively. The displacement rate during the first diffusion phase, $\Delta \bar{x}_{1}^{2}$ (blue dashed curve), is a small fraction of $r_{\mathrm{L}}^{2}$, thus indicating that the decoupling of the particle from its field line is a slow process compared to the particle gyration. The displacement $\Delta \bar{x}_{1}^{2}$ depends strongly on the turbulence amplitude, roughly as $\left(\delta B^{2} / B^{2}\right)^{2}$.

The displacement rate during the second diffusion, $\Delta \bar{x}_{2}^{2}$, multiplied by $10^{-3}$ in Figure 3(b) (solid green curve), is 3-4 orders of magnitude larger than $\Delta \bar{x}_{1}^{2}$ and of order $r_{\mathrm{L}}^{2}$, which indicates that at timescales $\gtrsim t_{2}$ the particles are fully separated from their initial field lines. The displacement $\Delta \bar{x}_{2}^{2}$ is roughly proportional to $\delta B / B$, similar to the dependence of the field-line diffusion coefficient on the turbulence amplitude in 2D turbulence (Matthaeus et al. 1995). The decrease of $\Delta \bar{x}_{2}^{2}$ from the $\delta B / B$ trend at large $\delta B^{2} / B^{2}$ can be caused by more efficient parallel scattering, as can be seen in, e.g., the nonlinear guiding center theory (Matthaeus et al. 2003).

Figure 3(c) shows the onset time of the transition phase, $t_{1}$, and the onset time of the second diffusion phase, $t_{2}$, with dashed blue and solid green curves, respectively, as a function of $\delta B^{2} / B^{2}$. Both onset times show a $\left(\delta B^{2} / B^{2}\right)^{-1}$ dependence on turbulence amplitude, with $t_{2} \sim 10 t_{1}$.

We also calculate the parallel scattering timescale, $\tau_{\|}=\lambda_{\|} / v$ (black dotted curve), where $\lambda_{\|}$is the scattering mean free path, 

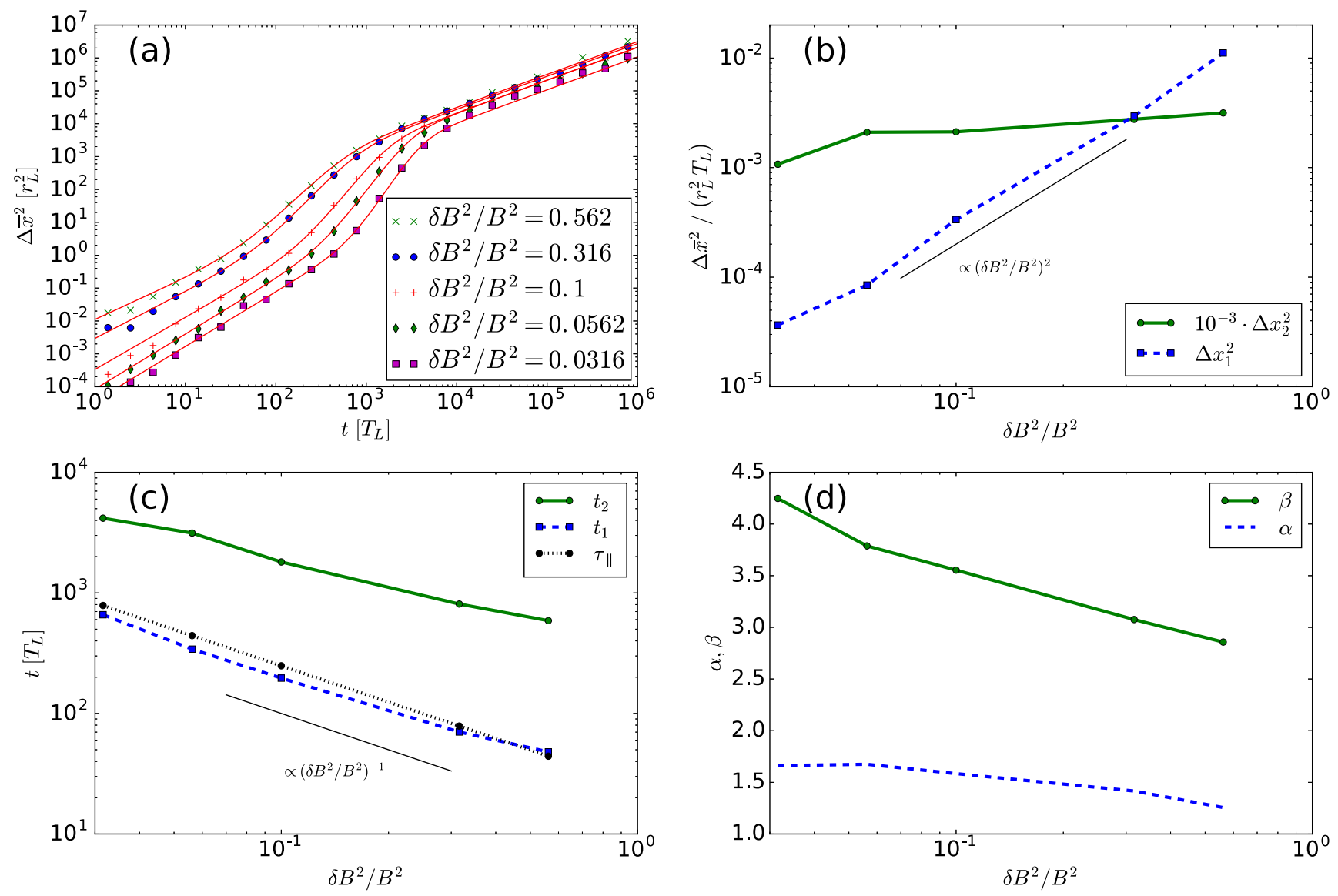

Figure 3. (a) Median displacement, in units $r_{\mathrm{L}}^{2}$, vs. time for five different $\delta B^{2} / B^{2}$, and the corresponding fit fo Equation (6). (b) $\Delta \bar{x}_{1}^{2}$ (dashed blue curve) and $10^{-3} \cdot \Delta \bar{x}_{2}^{2}$ (solid green curve) as a function of $\delta B^{2} / B^{2}$. (c) Transition times $t_{1}$ (dashed blue curve) and $t_{2}$ (solid green curve), and the parallel scattering timescale (black dotted curve), as a function of $\delta B^{2} / B^{2}$. (d) Power-law indices $\alpha$ (dashed blue curve) and $\beta$ (solid green curve) as a function of $\delta B^{2} / B^{2}$. The thin black lines in panels (b) and (c) depict the trend lines discussed in the text.

obtained as

$$
\lambda_{\|}=\frac{3 v}{8} \int_{-1}^{1} \frac{\left(1-\mu^{2}\right)^{2}}{D_{\mu \mu}} d \mu
$$

where $D_{\mu \mu}$ is the quasi-linear pitch angle diffusion coefficient (e.g., Jokipii 1966), calculated assuming that only the slab turbulence contributes to the parallel scattering of the particles. For the Kolmogorov turbulence spectral shape used in this study, the parallel scattering time depends on the turbulence amplitude and particle's Larmor radius as $\tau_{\|} \propto\left(\delta B_{\|}^{2} / B^{2}\right)^{-1} r_{\mathrm{L}}^{-2 / 3}$. As shown in Figure 3(c), $\tau_{\|}$values are close to the onset times of the transition phase, $t_{1}$. This implies that the decoupling process of particles from their field lines may be related to pitch angle scattering of the particles.

In Figure 3(d), we show the power-law indices of the first diffusion and the transition ranges $\alpha$ and $\beta$, with the dashed blue and solid green curves, respectively. The first diffusion range is superdiffusive, with $\alpha \sim 1.5$, showing an approach to the diffusive limit $\alpha=1$ for higher turbulence amplitudes. The transition phase (green curve) exhibits a very fast, superdiffusive cross-field expansion of the particle population from the initial magnetic field lines.

Figure 4 shows the median displacement versus time for proton energies $E=0.1,1$, and $10 \mathrm{MeV}$, with $\delta B^{2} / B^{2}=0.316$, in the same format as Figure 3. In panel (b) the rate of the displacement during the first diffusion (dashed blue curve) depends only weakly on the particle energy, with $\Delta \bar{x}_{1}^{2} / r_{\mathrm{L}}^{2} \propto v^{1 / 3}$. Likewise, panel (d) shows that the first diffusion range power-law index is nearly independent of the particle energy.

The second phase displacement rate, $\Delta \bar{x}_{2}^{2}$ (solid green curve in Figure 4 (b)), decreases as $\Delta \bar{x}_{2}^{2} / r_{\mathrm{L}}^{2} \propto 1 / v$, or $\Delta \bar{x}_{2}^{2} \propto v$. At the time-asymptotic limit (Equation (9)), the displacement thus behaves as $\Delta \bar{x}^{2}(t) \propto v t=s$, where $s$ is the distance a particle with velocity $v$ propagates in time $t$. Thus, the displacement of the particles during the second diffusion phase is a function of propagated distance, $s$, only. This indicates that the particle cross-field propagation during the second diffusion phase is dominated by the structure of the turbulent magnetic fields rather than the properties of the particles.

Panel (c) of Figure 4 shows the onset times $t_{1}$ and $t_{2}$ as a function of the particle energy, along with $\tau_{\|}$. The onset times scale with energy as $E^{-1 / 3}$, with $t_{2} \sim 10 t_{1}$. As can be seen, the first onset time $t_{1}$ is again very similar to the parallel scattering time, $\tau_{\|}$.

\section{DISCUSSION}

Our results show that the propagation of charged particles across the turbulently meandering field lines can be divided into three phases: the first diffusion, transition, and second diffusion phases. During the first diffusion phase, the particle displacement from the meandering field line grows 

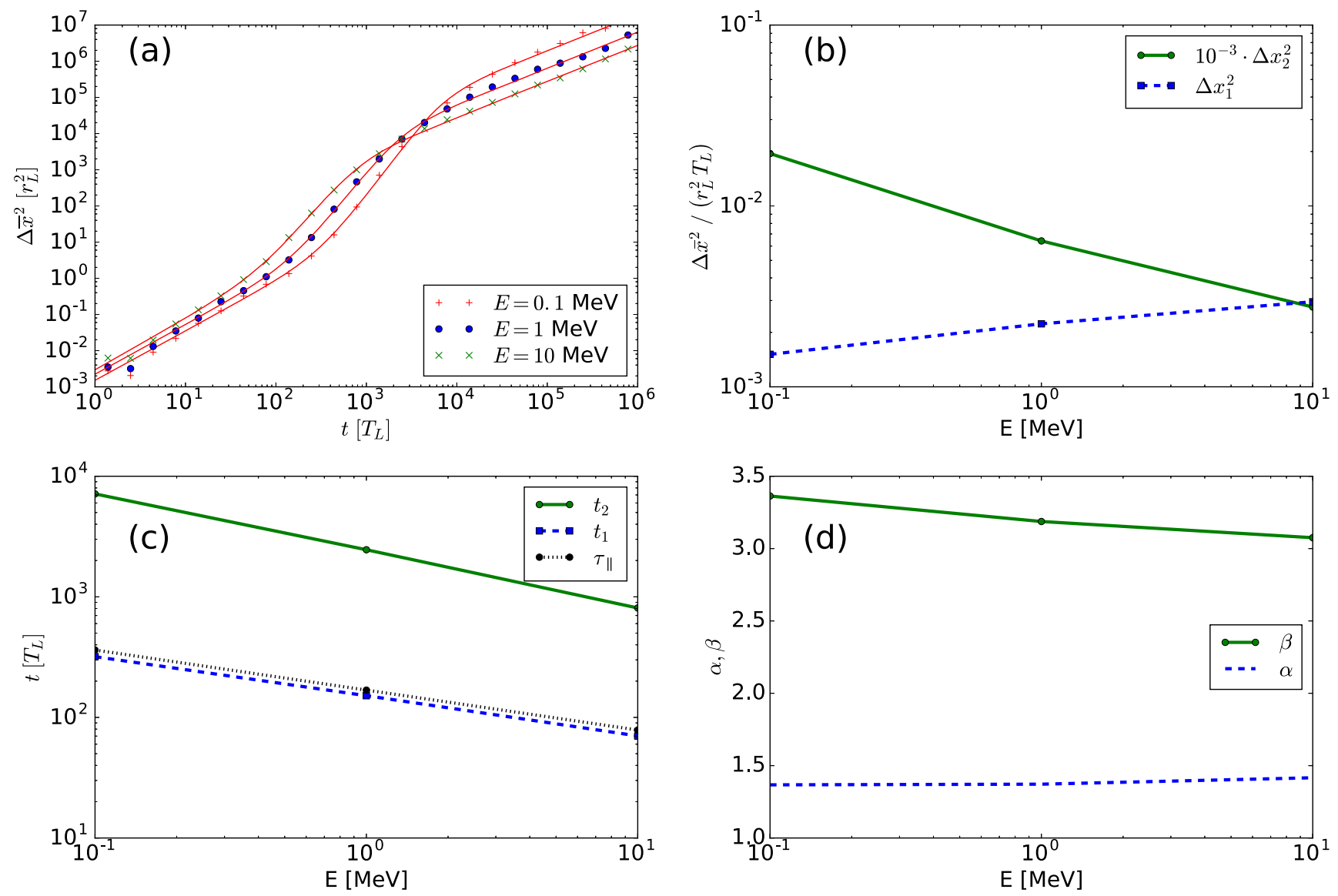

Figure 4. Median displacement vs. time for three different proton energies in turbulence with $\delta B^{2} / B^{2}=0.316$, and the corresponding fit fo Equation (6). The panels are as described in Figure 3.

superdiffusively, with the displacement rate increasing as a function of the turbulence amplitude and particle energy. As seen in Figures 3(a) and 4(a), however, during the first diffusion the displacement of the particles does not exceed the particle's Larmor radius scale, $r_{\mathrm{L}}$.

Thus, the particle can be considered as following a single field line during the first diffusion phase.

It is important to note that this does not imply inhibited particle propagation across the mean magnetic field during the first diffusion phase. Rather, the propagation of a particle across the mean magnetic field is determined by its propagation along a single meandering field line. The random walk of the field line can cause rapid, nondiffusive propagation of particles across the mean field direction (Laitinen et al. 2013). Thus, the particle propagation during the first diffusion phase follows a scenario depicted in the left panel of Figure 5. Recently, Laitinen et al. (2016) showed that such a fast cross-field transport of particles offers an explanation to fast and wide SEP events with realistic interplanetary conditions already with narrow source regions.

The first diffusion phase continues until the transition phase onset, $t_{1}$, which is of the order of tens to hundreds of gyroperiods for the particle and turbulence parameters used in this study. For a $10 \mathrm{MeV}$ proton in $\delta B^{2} / B^{2}=0.1$ turbulence, this corresponds to $0.75 \mathrm{hr}$, a time in which a particle beam would propagate a distance of $0.7 \mathrm{au}$. This implies that the first diffusion range is very significant for the early propagation of SEPs in the heliosphere. It should be noted that $t_{1}$ is much larger than the cross-field velocity correlation time obtained
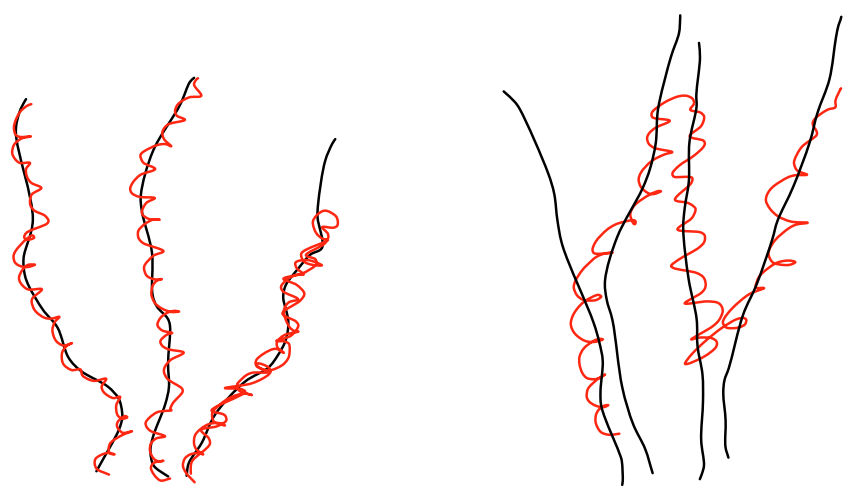

Figure 5. Schematic view of forming of the early (left) and late (right) diffusion phases, with the black curves depicting field lines and the red curves depicting particle orbits. In the early phase (left panel), particles remain close to their original field lines and spread across the mean field direction due to the random walk of the field lines. In the late phase (right panel), a particle decouples from a field line to follow another field line and, as a result, propagates across both the mean field and the individual meandering field lines.

from particle simulations by Fraschetti \& Giacalone (2012). However, their method yields the decorrelation time of a particle from an unperturbed orbit in a uniform magnetic field, whereas our method yields the decoupling timescale of the particle from a meandering field line.

As shown in Figures 3(c) and 4(c), the onset of the transition phase, $t_{1}$, is close to the parallel scattering timescale of the particles, $\tau_{\|}$, for the analyzed 2D-dominated turbulence cases. This could be interpreted as evidence for a strong link between 
the parallel scattering of the particles and the particle decoupling from the meandering field lines. However, the interpretation cannot be made quite so straightforwardly. In our simulations, the particles, all initiated with $v_{z}>0$, have all necessarily experienced pitch angle scattering to have $v_{z}<0$, required for them to return back to the plane $z=z_{0}$. Thus, during the first diffusion, all of the simulated particles have experienced pitch angle scattering from the positive to the negative pitch angle cosine hemisphere even if their propagation time is much smaller than $\tau_{\|}$. Therefore, while Figures 3(c) and 4(c) indicate that the onset time of the transition phase, $t_{1}$, is close to the parallel scattering time, $\tau_{\|}$, the connection between the parallel scattering and the particle decoupling from their field lines is likely more complicated than an effect due to backscattering of the particles.

The transition phase is rapid and strongly superdiffusive and continues until the onset of the second diffusion phase, $t_{2}$. As shown in Figures 3(c) and 4(c), the onset time of the second diffusion scales as $t_{2} \sim 10 t_{1}$, independent of particle energy and turbulence amplitude. If we consider the time $t_{1}$ as the timescale of the decoupling of the particle from its field line, the constant ratio $N=t_{2} / t_{1} \sim 10$ can be interpreted as the number of decouplings taking place until the asymptotic diffusive behavior in the second diffusion range is reached. In this interpretation, $t_{1}$ can be considered as the characteristic timescale, the "scattering time," for the particle diffusion across the mean magnetic field line. The scenario of subsequent decoupling of a particle from field lines leading to particle transport across the mean field is depicted in the right panel of Figure 5.

The transition to the second diffusion phase can be related to the recovery of diffusion reported by Qin et al. (2002), who studied the cross-field displacement of particles at all $z$ instead of the particles that have returned to $z=z_{0}$ (our method). They noted in their simulations that after an initial fast cross-field spreading, the running diffusion coefficient decreased, indicating subdiffusion, after which it reached a second diffusion phase. The fast spreading seen in the Qin et al. (2002) analysis can be understood as particles spreading in space along the meandering field lines as depicted in the left panel in our Figure 5 and the subdiffusion due to particles backscattering along the meandering field lines (compound diffusion; see, e.g., Kóta \& Jokipii 2000, and references therein). In our simulations, this behavior is depicted by particles remaining in the first diffusion phase, which we have quantified in this study. The second diffusion in Qin et al. (2002) is likely caused by particles decoupling from their field line (right panel in Figure 5), which releases the particles from the original field lines to trace the diffusive pattern of the turbulently meandering field lines.

As discussed in Section 3, the dependence of the second phase displacement rate, $\Delta \bar{x}_{2}^{2}$, on both the turbulence amplitude and energy is consistent with the particles diffusing across the mean field direction in a similar manner as the magnetic field lines diffuse. Thus, our results are consistent with the recent works that derive the time-asymptotic cross-field diffusion coefficients using the statistics of the field-line diffusion in the derivation (e.g., Matthaeus et al. 2003; Shalchi 2010; Ruffolo et al. 2012). However, the second diffusion is reached only at $t_{2}$, which is of the order of hundreds to thousands of gyroperiods. For a $10 \mathrm{MeV}$ proton in a $\delta B^{2} / B^{2}=0.1$ turbulence, this corresponds to $t_{2}=7.5 \mathrm{hr}$. Thus, our results suggest that the use of particle transport models where the cross-field diffusion coefficients are derived at the timeasymptotic limit cannot be justified when modeling the early propagation of SEPs in the interplanetary space.

It should be noted that the particles being decoupled from their field lines do not imply that a solution of a diffusive particle transport equation can be used to describe the particle distribution everywhere in space. As shown in Laitinen et al. (2013), the particles at 1 au from the injection site spread to a wide cross-field range early in the event due to field-line meandering. While the particles decouple from the field lines at timescale $t_{2}$, the cross-field extent of the particles at 1 au is still dominated by the initial spread of the particles along meandering field lines. As shown in Figure 3 of Laitinen et al. (2013), the $10 \mathrm{MeV}$ proton spreading due to decoupling results in time-asymptotic diffusion behavior at 1 au only $\sim 20 \mathrm{hr}$ after their injection in turbulence with $\delta B^{2} / B^{2}=0.1$ turbulence.

To understand when the particle propagation can be considered as time-asymptotic, we must understand how the decoupling of the particles takes place and how it contributes to the transition to the time-asymptotic propagation phase. Recently, Fraschetti \& Jokipii (2011) studied the decoupling of particles from the field lines by deriving a diffusion coefficient for cross-field propagation of particles due to curvature and gradient drifts caused by the turbulent magnetic fields. Their first-order analysis found no contribution from the 2D turbulence to decoupling of particles from the field lines, whereas slab turbulence resulted in subdiffusive decoupling. Thus, their result is not consistent with our findings, where in the slab+2D turbulence the returning particles spread from their field lines superdiffusively, as shown in Figures 3 and 4.

The decoupling of particles from their field lines may also be related to how the field lines decouple from each other. Ruffolo et al. (2004) found that neighboring field lines initially follow each other almost coherently, with slow diffusive divergence that turns into a fast spreading at length scale $l_{g}$, given by

$$
l_{g}=\frac{\lambda_{c}}{2} \frac{\delta B_{\|}^{2}}{\delta B_{\perp}^{2}}
$$

where $\lambda_{c}$ is the parallel correlation length of the turbulence,

$$
\lambda_{c}=\frac{\pi}{2} \frac{P_{\text {slab }}\left(k_{\|}=0\right)}{\delta B_{\|}^{2}} \approx 0.79 L_{c} .
$$

In the simulations presented in Figures 3 and 4, however, $\delta B_{\|}^{2} / \delta B_{\perp}^{2}=1 / 4$; thus, the neighboring field lines would decorrelate already at a fraction of parallel correlation length, thus much shorter than $v t_{1}$ given by our simulations.

To further study whether the Fraschetti \& Jokipii (2011) and Ruffolo et al. (2004) formulations can be applied to our results, we ran additional simulations with slab-dominated turbulence. It should be noted that cross-field propagation of charged particles is strongly inhibited in pure slab turbulence (Jokipii et al. 1993; Jones et al. 1998). In addition, the field-line separation scale, $l_{g}$, as defined by Equation (11), would be infinite in pure slab turbulence, indicating the absence of strong field-line separation. Thus, as we are interested in understanding non-negligible cross-field propagation of particles in turbulent magnetic fields, we use slab-dominated turbulence with a small 2D component instead of pure slab turbulence. Such a slab-dominated turbulence mix allows for finite $l_{g}$, and 


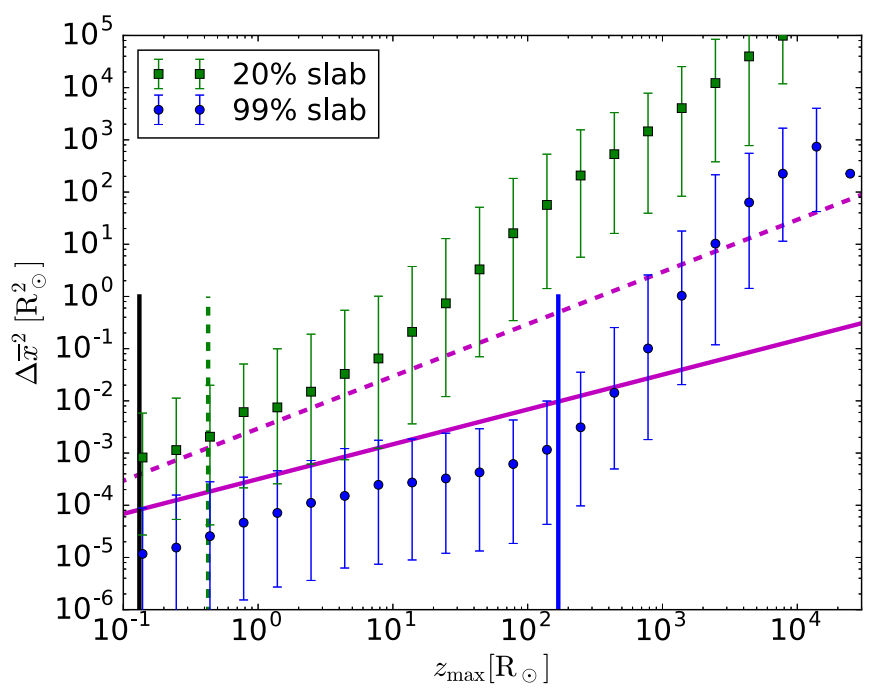

Figure 6. Mean displacement as a function of maximum distance along mean field direction for different slab turbulence energy fractions, with the error bars depicting the upper and lower deciles. For both cases, $\delta B_{\|}^{2} / B^{2}=0.112$ and $E=10 \mathrm{MeV}$. The vertical black line shows the gyroradius of the particle. The solid blue and dashed green vertical lines give the Ruffolo et al. (2004) fieldline divergence scale $l_{g}$ for the slab- and 2D-dominated cases, respectively. The solid and dashed magenta lines show the Fraschetti \& Jokipii (2011) result for slab and isotropic turbulence, respectively.

particle propagation is not as constrained as in pure slab turbulence.

In Figure 6, we show results of simulations of $10 \mathrm{MeV}$ protons in turbulence with $\delta B_{\|}^{2} / B^{2}=0.112$ for $20 \%$ (green squares) and $99 \%$ (blue circles) slab contributions, with the error bars representing the lower and upper deciles, respectively. We present the displacement $\Delta \bar{x}^{2}$ as a function of a length scale instead of time, to gain understanding of the transition process in terms of $l_{g}$. As length scale, we consider the maximum distance the particle has propagated along the mean field direction, $z_{\max , i}=\max \left\{\left|z_{i}-z_{0 i}\right|\right\}$, before returning to the plane it was injected at. In our analysis, we have used mean instead of median square displacement, to obtain better correspondence with the displacement values predicted by the Fraschetti \& Jokipii (2011) theory. It should be noted, though, that as we simulate the particles only until their first time of return to the $z=z_{0}$ plane, full correspondence with the absolute values cannot be expected.

In addition to the mean displacement of the returning particles, we show in Figure 6 the field line divergence scale $l_{g}$ (Equation (11)) with the vertical solid blue line and dashed green line for the slab- and 2D-dominated cases, respectively, and the particle Larmor radius scale with the vertical black line. The $\Delta x^{2}$ due to stochastic drifts for slab turbulence, as given by Fraschetti \& Jokipii (2011), is shown with the solid magenta curve. It should be noted that our simulations are not sufficiently long for analyzing the second diffusion range by fitting Equation (6) in the slab-dominated case, due to computational limitations. Thus, we will concentrate below on analysis of the first diffusion range and the onset of the transition phase and will discuss the transition phase only qualitatively.

As can be seen in Figure 6, the first diffusion range and the subsequent transition phase can be observed for both the slabdominated and 2D-dominated turbulence. For the slabdominated turbulence, the subdiffusive trend of the Fraschetti
\& Jokipii (2011) result (solid magenta line) is well replicated by our simulations (blue circles). Thus, in the parameter range relevant to the Fraschetti \& Jokipii (2011) work, at scales $\ll l_{g}$, where the field-line separation due to the $1 \% 2 \mathrm{D}$ component is negligible, we find agreement with the previous theoretical work and our results. The superdiffusive displacement increase in the 2D-dominated turbulence (green squares in Figure 6) seen in our simulations, however, deviates strongly from the Fraschetti \& Jokipii (2011) result. We suggest two possible explanations for this. One is related to the field-line divergence scale $l_{g}$. As shown by the dashed green vertical line in Figure 6, $l_{g} \sim 3 r_{\mathrm{L}}$ for the 2D-dominated case, well below the start of the transition from the first diffusion range, at $z_{\max } \sim 10 R_{\odot}$. Thus, the field-line decoherence at short length scales may influence the particle spreading already during the first diffusion range, possibly turning the subdiffusive spreading predicted by Fraschetti \& Jokipii (2011) (solid magenta curve) into the superdiffusive behavior shown in Figure 6.

On the other hand, as speculated by Fraschetti \& Jokipii (2011), the particle decoupling may be affected by secondorder effects by the 2D turbulence component, which their theory does not account for. Fraschetti \& Jokipii (2011) present their result also for isotropic turbulence, shown with a dashed magenta line in Figure 6. As can be seen, it matches the simulation trend and level of the 2D-dominated case considerably better than their slab result.

As shown in Figure 6, the transition from the first diffusion to the transition range in the slab-dominated case (blue circles) takes place for particles that have reached the distance $z_{\max } \sim l_{g}=168 R_{\odot}$. Thus, in the slab-dominated turbulence, the strong separation of the field lines at scales $l_{g}$ (Ruffolo et al. 2004) appears to be connected to the particle decoupling from their field lines. A similar conclusion cannot be drawn in the 2D-dominated case (the green squares in Figure 6): as suggested by the dashed green vertical line, the field lines are strongly separated much before the onset of the transition phase, at around $z_{\max } \sim 10 R_{\odot}$.

Figures 3(a) and 4(a) suggest a different explanation for the transition of the particle displacements from the first diffusion range in the $2 \mathrm{D}$-dominated turbulence. The displacement can be seen to reach the magnitude of the particle Larmor radius, $r_{\mathrm{L}}$, at the transition onset time, $t_{1}$, in all of our simulations with 2D-dominated turbulence. We quantify this in Figure 7 , where we compare the displacement of the particle at the transition onset time, i.e., $\Delta \bar{x}^{2}\left(t_{1}\right)$ (solid green curve), for different simulations, with the particle Larmor radius $r_{\mathrm{L}}$ (dashed blue curve). As can be seen, at the onset of the transition range, the particles have moved away by an order of Larmor radius from their original gyrocenter. It should be noted that in the slabdominated case (Figure 6), the mean displacement is considerably smaller than the Larmor radius at the time of the transition, at around $z_{\max } \sim 200 R_{\odot}$. Thus, the decoupling of the particles from their field lines, as defined by the change from the first diffusion phase to the superdiffusive transition phase at $t_{1}$, may be fundamentally different in slab- and 2Ddominated turbulence.

The role of the particle's Larmor radius as a determining factor for particle cross-field propagation has been discussed in the context of electron heat transport in tokamak plasmas by Rechester \& Rosenbluth (1978), who considered the electrons to be displaced from their field lines by Coulomb collisions. Ruffolo et al. (2012) used a similar idea to model the time- 

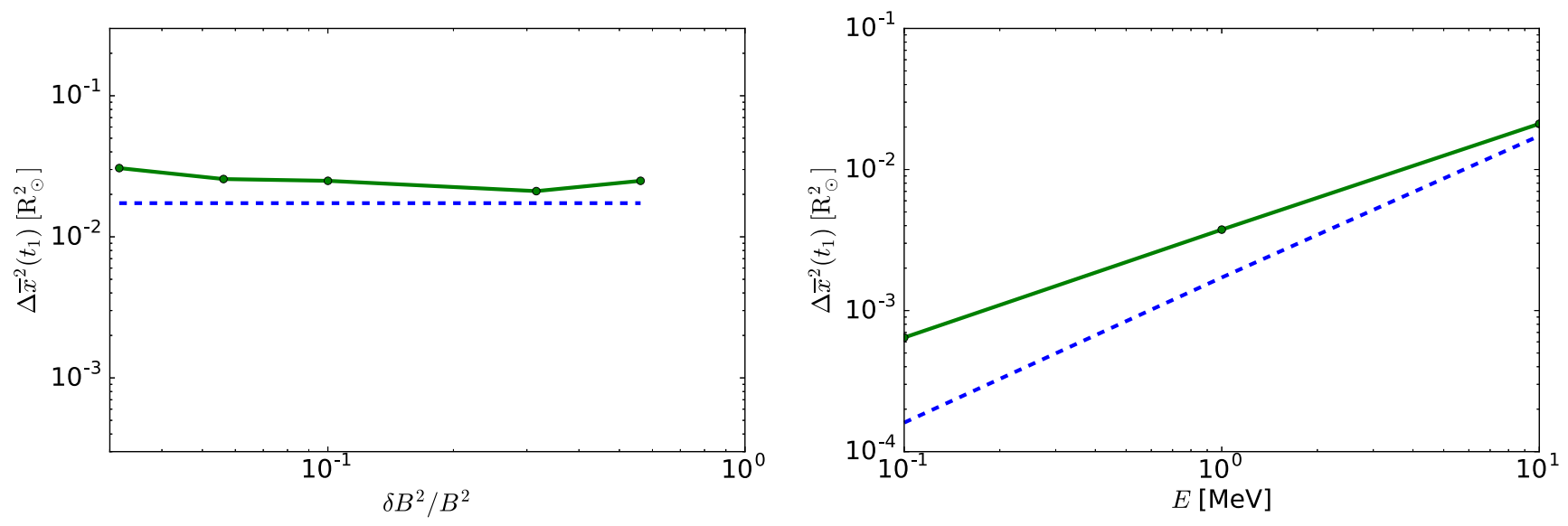

Figure 7. Median displacement of the particles at time $t_{1}, \Delta \bar{x}^{2}\left(t_{1}\right)$ (solid green curve). The dashed blue line shows the square of the particle gyroradius.

asymptotic diffusion of particles in turbulent plasmas as ballistic propagation of particles along meandering field lines, punctuated by decouplings on the timescale of the parallel scattering time of the particles. This concept is supported by the close similarity of the transition phase onset time $t_{1}$ and the parallel scattering timescale $\tau_{\|}$seen in the 2D-dominated turbulence cases of our study. However, as discussed above, the particles analyzed in this study have all backscattered in field-parallel direction to return to the initial $z=z_{0}$ plane. Thus, during the first diffusion, the parallel scattering does not efficiently decouple particles from their initial field lines.

In addition, the similarity of $t_{1}$ and $\tau_{\|}$is valid only for the 2D-dominated cases analyzed in this study. For the slabdominated scenario we determined $t_{1} \sim 1000 T_{\mathrm{L}}$, considerably larger than the parallel diffusion time, $\tau_{\|}=44 T_{\mathrm{L}}$ for $\delta B_{\|}^{2} / B^{2}=0.112$ and the proton energy of $10 \mathrm{MeV}$. Therefore, our results indicate that scattering alone does not imply a significant displacement of particles from their field lines.

Pitch angle scattering may, however, be significant for crossfield propagation of particles in turbulent magnetic fields. As discussed in Section 3, the transition onset time $t_{1}$ depends on the turbulence amplitude and energy in a similar way to the pitch angle diffusion timescale $\tau_{\|}$, suggesting that faster pitch angle isotropization leads to faster transition onset $t_{1}$. This may be connected to the proportionality of the cross-field diffusion due to stochastic drifts on $\left(1-\mu^{2}\right)^{2}$ (Fraschetti 2016), where $\mu$ is the pitch angle cosine. This dependence indicates that an isotropic distribution would decouple from the meandering field lines faster than an anisotropic one.

It should be noted that in view of the schematic picture presented in the right panel of Figure 5, the dependence of the cross-field particle diffusion on $\mu$ is not obvious. Strong crossfield propagation due to field-line meandering requires efficient decoupling of particles from their field lines and, in light of the Fraschetti (2016) result, pitch angles $\sim \pi / 2$. On the other hand, the spreading of particles across the mean magnetic field direction due to propagation along meandering field lines requires large particle velocities along the field lines, i.e., $|\mu| \sim 1$. Thus, pitch angle dependence of the cross-field particle diffusion particle transport may be more complicated than the recently discussed proportionality to $|\mu|$ or $\left(1-\mu^{2}\right)$ (see, e.g., Dröge et al. 2010; Qin \& Shalchi 2014; Strauss \& Fichtner 2015, and discussion therein). Overall, our simulations show the importance of understanding the microphysics of the particle decoupling from their original field lines for understanding the propagation of particles across the mean magnetic field in turbulent plasmas.

\section{CONCLUSIONS}

In this work, we have studied how charged particles spread across the mean field direction in turbulent magnetic fields superimposed on a uniform field, by analyzing the displacement of a particle from its initial, meandering, field line instead of the mean field. Our results show the following:

1. The particles initially follow their initial meandering field lines, over timescales $t_{1} \sim 50-700 T_{\mathrm{L}}$, or 10-150 minutes, for $10 \mathrm{MeV}$ protons in turbulent magnetic fields corresponding to the solar wind at $1 \mathrm{au}$ from the Sun, with $\delta B^{2} / B^{2}=0.05-0.5$ (Burlaga \& Turner 1976; Bavassano et al. 1982).

2. The time-asymptotic diffusion, consistent with cross-field diffusion dominated by random walk of field lines, is reached in timescales $t_{2} \sim 10 t_{1}$, or hours to a day for a $10 \mathrm{MeV}$ proton.

3. The transition from the first to the second diffusion range may depend on stochastic gradient and curvature drifts, field-line decoherence, and pitch angle scattering of the particles, depending on the turbulence parameters.

Our results suggest that the first diffusion range, where the particles stay on their field lines, is significant for SEP event evolution: $10 \mathrm{MeV}$ protons simulated propagate a distance of 1 au in $\sim 60$ minutes, which is of the order of the timescale a particle remains completely bound to its field, $t_{1}$, in turbulence conditions corresponding to those of the solar wind at $1 \mathrm{au}$. Further, full relaxation to the time-asymptotic diffusive particle propagation would be reached in a timescale of hours to a day. It should be noted that the turbulence parameters vary radially (e.g., Bavassano et al. 1982) and also as a function of time (e.g., Burlaga \& Turner 1976). The temporal variation will cause also longitudinal variation of the turbulence parameters, due to the solar rotation. Also, the large-scale magnetic field structure and the associated large-scale particle drifts (e.g., Marsh et al. 2013) may influence the particle decoupling from their field lines. Thus, a full study, including utilizing solar wind and turbulence observations and models, is required to understand the SEP propagation in the interplanetary space in different solar wind turbulence conditions.

Our results indicate that the field-line meandering controls the particle propagation both in the early phases and at time- 
asymptotic phases of particle cross-field spreading. However, as depicted in the schematic view in Figure 5, the effect of the field-line meandering manifests itself completely differently at these phases. The early cross-field propagation is characterized by particles following their initial field lines and is thus deterministic propagation along stochastic paths. The timeasymptotic propagation, on the other hand, is characterized by particles decoupling from their original field lines at timescales $t_{2}$, which causes the particles to random walk from one random-walking field line to another.

The mechanism of the early time particle cross-field propagation presented in our study provides also a possible explanation for the SEP intensity dropouts. These dropouts, observed in some SEP events (e.g., Mazur et al. 2000), imply strong cross-field gradients in spatial SEP distribution in these events. While such gradients would be smoothed by the timeasymptotic cross-field diffusion (Dröge et al. 2010; Wang et al. 2014), our simulations show that the cross-field propagation of the particles across the meandering field lines is negligible during the first diffusion phase, enabling the intensity dropouts to persist.

The mechanism behind the transition phase between the first and second diffusion ranges remains unclear and requires further study. Our results show that the first diffusion phase and the transition phase of the particle cross-field transport exist both for $2 \mathrm{D}$ and slab-dominated turbulence, which suggests that the transition is a general feature in early cross-field propagation of particles in turbulent magnetic fields. We have identified potential mechanisms through comparison with the stochastic drift diffusion theory (Fraschetti \& Jokipii 2011), the field-line decoherence (Ruffolo et al. 2004), the parallel scattering timescale of the particles, and the distance the particle deviates from its field line before the transition commences. However, the relative contribution of different processes appears to depend on the composition of the turbulence. In a future work, we will study the interplay between different phenomena contributing to particle crossfield propagation by using guiding center simulations that include the relevant physics and comparing them with full-orbit simulations, as well as theoretical results. Such a study would be capable of improving our understanding on not only the transition stage of the charged particle cross-field propagation but also how the time-asymptotic diffusive behavior is formed.
T.L. and S.D. acknowledge support from the UK Science and Technology Facilities Council (STFC) (grants ST/ J001341/1 and ST/M00760X/1) and the International Space Science Institute as part of international team 297. Access to the University of Central Lancashire's High Performance Computing Facility is gratefully acknowledged.

\section{REFERENCES}

Bavassano, B., Dobrowolny, M., Mariani, F., \& Ness, N. F. 1982, JGR, 87,3617

Burger, R. A., Potgieter, M. S., \& Heber, B. 2000, JGR, 105, 27447

Burlaga, L. F., \& Turner, J. M. 1976, JGR, 81, 73

Dresing, N., Gómez-Herrero, R., Klassen, A., et al. 2012, SoPh, 281, 281

Dröge, W., Kartavykh, Y. Y., Dresing, N., Heber, B., \& Klassen, A. 2014 JGRA, 119, 6074

Dröge, W., Kartavykh, Y. Y., Klecker, B., \& Kovaltsov, G. A. 2010, ApJ, 709,912

Fraschetti, F. 2016, ASTRP, 2, 63

Fraschetti, F., \& Giacalone, J. 2012, ApJ, 755, 114

Fraschetti, F., \& Jokipii, J. R. 2011, ApJ, 734, 83

Giacalone, J., \& Jokipii, J. R. 1999, ApJ, 520, 204

Giacalone, J., \& Jokipii, J. R. 2012, ApJL, 751, L33

Gray, P. C., Pontius, D. H., \& Matthaeus, W. H. 1996, GeoRL, 23, 965

He, H.-Q., Qin, G., \& Zhang, M. 2011, ApJ, 734, 74

Jokipii, J. R. 1966, ApJ, 146, 480

Jokipii, J. R., Kota, J., \& Giacalone, J. 1993, GeoRL, 20, 1759

Jones, F. C., Jokipii, J. R., \& Baring, M. G. 1998, ApJ, 509, 238

Kóta, J., \& Jokipii, J. R. 2000, ApJ, 531, 1067

Laitinen, T., Dalla, S., \& Marsh, M. S. 2013, ApJL, 773, L29

Laitinen, T., Kopp, A., Effenberger, F., Dalla, S., \& Marsh, M. S. 2016, A\&A, 591, A18

Marsh, M. S., Dalla, S., Kelly, J., \& Laitinen, T. 2013, ApJ, 774, 4

Matthaeus, W. H., Gray, P. C., Pontius, D. H., Jr., \& Bieber, J. W. 1995, PhRvL, 75, 2136

Matthaeus, W. H., Qin, G., Bieber, J. W., \& Zank, G. P. 2003, ApJL, 590, L53

Mazur, J. E., Mason, G. M., Dwyer, J. R., et al. 2000, ApJL, 532, L79

Minnie, J., Matthaeus, W. H., Bieber, J. W., Ruffolo, D., \& Burger, R. A. 2009, JGRA, 114, a01102

Parker, E. N. 1965, P\&SS, 13, 9

Potgieter, M. S., Vos, E. E., Boezio, M., et al. 2014, SoPh, 289, 391

Qin, G., Matthaeus, W. H., \& Bieber, J. W. 2002, ApJL, 578, L117

Qin, G., \& Shalchi, A. 2014, ApPhR, 6, 1

Qin, G., Wang, Y., Zhang, M., \& Dalla, S. 2013, ApJ, 766, 74

Rechester, A. B., \& Rosenbluth, M. N. 1978, PhRvL, 40, 38

Ruffolo, D., Matthaeus, W. H., \& Chuychai, P. 2004, ApJ, 614, 420

Ruffolo, D., Pianpanit, T., Matthaeus, W. H., \& Chuychai, P. 2012, ApJL, 747, L34

Shalchi, A. 2010, ApJL, 720, L127

Strauss, R. D., \& Fichtner, H. 2015, ApJ, 801, 29

Wang, Y., Qin, G., Zhang, M., \& Dalla, S. 2014, ApJ, 789, 157

Zhang, M., Qin, G., \& Rassoul, H. 2009, ApJ, 692, 109 